\title{
Balancing the Requirements for a Zero False Positive/Negative Forward Collision Warning
}

\author{
Natalya An*, Michael Maile ${ }^{\dagger}$, Daniel Jiang ${ }^{\dagger}$, Jens Mittag* and Hannes Hartenstein* \\ ${ }^{*}$ Institute of Telematics and Steinbuch Centre for Computing, Karlsruhe Institute of Technology \\ Email: \{natalya.an, jens.mittag, hannes.hartenstein\}@kit.edu \\ ${ }^{\dagger}$ Mercedes-Benz Research \& Development North America, Inc. \\ Email: \{michael.maile, daniel.jiang\}@daimler.com
}

\begin{abstract}
The main benefit of cooperative vehicular communication networks is the increased traffic safety that safety applications could introduce. Although the technological aspects have been extensively researched, the feasibility regions in which communication could support safety applications are yet not clear. This is, primarily, due to the lack of clearly defined application requirements. Moreover, reduction of false positives and false negatives are either not considered at all or only partially. In the current paper we investigate under which conditions vehicular communication can support safety applications. For this, we identified the requirements of safety applications, on the example of Forward Collision Warning, first from application perspective and pursuing zero false positive and zero false negative constraint. Afterwards, we quantify what is the maximum vehicle density for which application requirements can be supported under realistic communication conditions, as well as how much the idealistic application requirements have to be relaxed in order to achieve a balance between scalability and zero false rates.
\end{abstract}

\section{INTRODUCTION}

Using wireless communication technologies to exchange information among vehicles on the road has been studied over the past decades, in particular with a focus on safetyrelated applications. While the efforts carried out are essential to understand the limits of the communication system, a further step is required to answer the question whether the communication system is able to successfully support the envisioned safety applications. For this, the requirements of safety applications need to be clearly defined. Application requirement analysis, carried out in the past, often does not consider the minimization of false positives, i.e. warning when it is not needed, and minimization of false negatives, i.e. missing of dangerous situations. However, small false positive and false negative rates are essential for success and acceptance of safety applications

In this paper we first identify the exact requirements of a Forward Collision Warning (FCW) safety application from application perspective by explicitly pursuing zero false positive and zero false negative constraints. We perform a requirement analysis with the idealistic assumptions: human factors (e.g., driver's reaction time) are known precisely, GPS position estimation is error-free and communication is perfect, i.e., all the packets that are sent, are being immediately received. Afterwards, we indicate to which degree realistic communication could support the application, i.e. we indicate the maximum vehicle density for which the FCW application can be supported. We also point out how much strict requirements have to be relaxed to achieve a balance between scalability and zero false rates.

The paper is structured as follows: In Section II we outline the related work. In Section III we discuss how we model the FCW application and our method to identify its requirements. Section IV discusses the application requirements analysis. Subsequent implications from realistic traffic conditions are addressed in Section V. In the last two Sections VI and VII we present the impact of realistic communication conditions on application requirements and conclude the paper.

\section{RELATED WORK}

In general, application requirements are assumed as presented in [1] where only general suggestions for requirements are given, such as transmission frequency should be no less than $10 \mathrm{~Hz}$, effective communication range should be $\approx 100$ $300 \mathrm{~m}$ and maximum delay should not exceed $\approx 100-300 \mathrm{~ms}$. These values are not justified from application perspective yet, i.e., fulfillment of $100 \mathrm{~ms}$ delay requirement does not necessarily correspond to a collision-free driving.

The authors of [2] characterized application reliability and developed an analytical model to overcome the mismatch between application and communication performances. The application reliability is characterized as receiving at least one packet in a tolerance time window $T$. The time window $\mathrm{T}$ "represents" application requirement and is set to a value $0.3-3.0 \mathrm{~s}$, but these values are not justified from application perspective.

There are several works that express application requirements in a form of a certain distance at which communication has to be reliable in the sense of [2], meaning receiving at least one packet up to this distance during time window T. For example, the authors of [3] expressed application requirements as a critical distance, which is calculated based on kinematic equations and is enough to accomplish some driving maneuver without disturbing other vehicles. The parameter time window $\mathrm{T}$ is still not explicitly justified. The authors mention that reception of only one message is enough to avoid dangerous situation and that reception of a message at distance slightly larger and much larger than calculated distance produces the same application reliability. The single reception at larger 
distances might result in a false warning, as situation can still change and the warning perceived as unnecessary.

Novel application metrics have been also proposed in the literature e.g., update delay [4], number of invisible neighbors [5], information age [6] and position accuracy [7]. Although these metrics are one step forward in application requirements analysis, it is not clear whether, e.g., the number of invisible neighbors should be 0 and whether a position accuracy of below 1 meter is sufficient for a safe driving. Thus, there is a lack of clear and useful safety application requirements definition.

\section{SySTEM MODEL}

Prior to presenting the methodology for requirements analysis we require a clear definition of the application. Although, in this paper, we analyze a FCW application based on periodic updates, the general evaluation method is applicable to other applications based on periodic updates.

\section{A. Forward Collision Warning Description}

The design of the FCW application is mainly based on [8] and [9]. Some extra characteristics, as e.g., automatic braking, are added based on our own judgment, to make the application complete. The FCW application is "active" between two vehicles that are driving one after the other in the same direction and on the same lane. The FCW should warn the driver of the following vehicle $(\mathrm{FV})$ in case the approach to the leading vehicle (LV) is too fast, i.e., the rearend collision is impending if no braking is applied. In case the driver does not respond to a warning, the system should brake automatically in order to avoid a collision.

The FCW application distinguishes three states of operation: I - warning "off”, II - warning "on" and III - automatic braking. When the inter-vehicular distance between FV and $\mathrm{LV}$ is large, the FV is in the warning "off" state. If the inter-vehicular distance decreases, the FV enters the warning "on" state in which the FCW system presents a warning to a driver. If the inter-vehicular distance continues to decrease, the FV enters into an automatic braking state in which the FCW brakes automatically. Two critical inter-vehicular distances that separate the three states from each other are the Warning Distance $D_{W}$ and Automatic Braking Distance $D_{A B}$, see Fig. 1. The Warning distance is the inter-vehicular distance at which the driver of the FV should start decelerating with comfortable deceleration rate, in order to achieve the same speed as the LV while maintaining the desired safety distance. The warning distance should also account for the reaction time of the driver. If the driver of the FV fails to respond to a warning by braking, the system will start braking automatically at the Automatic Braking Distance. The $D_{A B}$ distance calculation does not contain a driver's reaction time delay and typically should use the maximum achievable deceleration rate of the vehicle ${ }^{1}$. In case the driver of the FV sufficiently

${ }^{1}$ A softer value for deceleration at $D_{A B}$ could be also used. We assume, if FCW application is fail safe, the automatic braking will happen only in a case of emergency and should also trigger transmission of a Decentralized Environmental Notification Message (DENM).

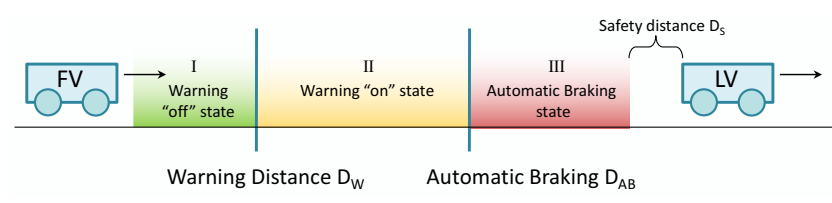

Fig. 1. The Forward Collision Warning Scenario. FV - following vehicle, LV - leading vehicle.

responds to a warning (by applying enough braking force) the system has to know when the FV will enter back to the warning "off" state, so it could stop warning the driver. The calculation of both critical distances should also consider the system delay between the time of decision to issue a warning and the time of a warning provided to the driver as well as between the time of decision to brake automatically and the time of actual braking.

\section{B. Methodology}

In our requirements analysis of safety applications we have two challenges: Zero False Positives and Zero False Negatives. False positive is when a warning is issued, but there is no collision threat. False positives are undesirable as the driver's trust in the system reduces with the increasing number of false alarms. In the automotive industry, the typical value of acceptable false positive ratio is less than $20 \%$ [9]. False negative is when a warning is not issued, but there is a collision threat. It is the core of safety applications to always warn when there is a collision threat and it should be done in a timely manner so that a driver could safely react. A typical acceptable false negative ratio is usually not stated.

Fig. 2 illustrates false positive and false negative alarms. For zero false positive and zero false negative the FCW application should only warn at $D_{W}$ and automatically brake only at $D_{A B}$. If a warning or an automatic braking happens before the critical distance, this is a false positive, if a warning or an automatic braking happens too late, this is a false negative.

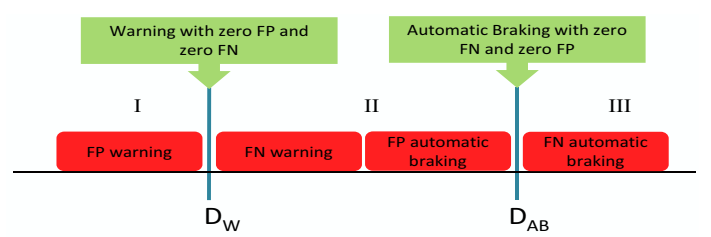

Fig. 2. False Positive (FP), False Negative (FN) alarms and FCW states.

Consider the example depicted in Fig. 3. In Fig. 3a) the FCW system of FV receives an update from LV and determines the two critical distances $D_{W}$ and $D_{A B}$ and operation states (I-III). If the system does not receive further updates and simply presents a warning at $D_{W}$ it might cause a false warning, as there is an uncertainty about the position of the LV since the last update. For example, the LV might accelerate and a new $D_{W}$ will now be shifted away from FV, see Fig. 3b). The related work mostly does not consider this possibility of false positive warning. If $\mathrm{LV}$ reduces its speed, the $D_{W}$ will be shifted closer to FV and the time that FV will spend in a warning "off" state will become shorter, see Fig. 3c). Not taking this into account might produce false negative warnings. 
a)

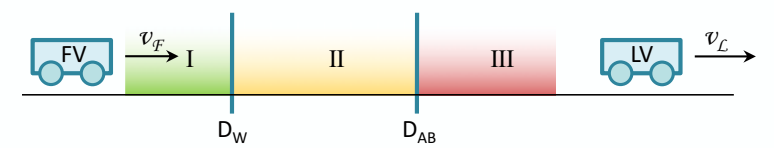

b)

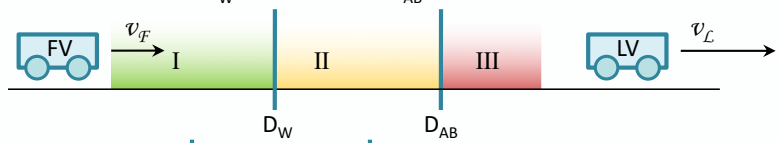

c)

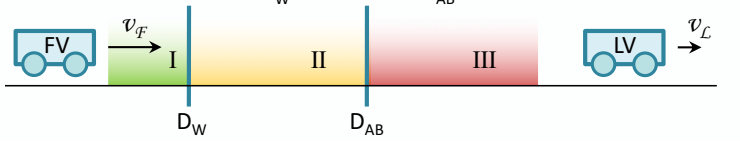

Fig. 3. Change in relative speed changes $D_{W}$ and $D_{A B}$ and FCW states.

As a FCW application depends on information received over wireless communication, we define the application requirements by answering the question: How far do vehicles need to communicate and when do vehicles need to receive a next update from other vehicles so that the application operates successfully, both with zero false positives and zero false negatives?

As a first step, we perform a requirement analysis with idealistic assumptions of perfect knowledge. Then we discuss implications imposed by realistic traffic and communications conditions.

\section{IDEALISTIC FCW REQUiREMENTS ANALYsis}

To answer the question stated in the previous section and thus to define application requirements we determine two main transmission parameters: transmission range and rate. Transmission range impacts how far vehicles can communicate and is determined by the largest of the two critical distances the warning distance $D_{W}$. Whereas transmission rate impacts how often vehicles can communicate and is determined by the earliest time after which the FV could change its state.

How far should vehicles communicate? According to the definition in Section III-A warning distance $D_{W}$ is the distance at which the following vehicle FV, which is moving with the speed $v_{F}$, should start applying deceleration $a_{F}$ in order to come to the same speed $v_{L}$ as LV. The safety distance $D_{S}$ and the distance traveled during reaction time $t_{R}$ and system delay $t_{\text {sys }}$ should be also taken into account. The derivation of the equation is based on basic kinematic equations and provided online on our website [10]. The resulting equation to calculate the warning distance $D_{W}$ is as follows:

$$
D_{W}=\frac{\left(v_{F}-v_{L}\right)^{2}}{2\left(a_{L}-a_{F}\right)}+\left(v_{F}-v_{L}\right) \cdot\left(t_{R}+t_{s y s}\right)+D_{S}
$$

where $v_{F}>v_{L}, a_{F}<0$ and acceleration of LV $a_{L}=0, \mathrm{LV}$ is either stopped or moving with a constant speed.

How often should vehicles communicate? Once FV determines its current state by receiving an update at some intervehicular distance $D$, the time until a possible state change determines the allowable delay $t_{\text {update }}$ to the next update. The state change will happen earlier if the speed difference $\Delta v$ is increased. We are looking for the maximum time $t_{\text {update }}$ during which vehicles might change their speed, in realistic boundaries, and the FV will still be in the same state as at the reception of the last update. As soon as the new inter-vehicle distance $D^{\text {new }}$ equals the new warning distance $D_{W}^{\text {new }}$, the next update is needed to make sure no false alarms are committed.

In other words, we are looking for $t_{\text {update }}$ for which the following equation is true:

$$
D^{\text {new }}=D_{W}^{\text {new }}
$$

The new inter-vehicular distance $D^{\text {new }}$ accounts for the distances that both FV and LV traveled during $t_{\text {update }}$ since the last update at $D$. The new warning distance $D_{W}^{n e w}$ is calculated as in Equation 1, but considers that the speed of vehicles can change. By substituting $D_{W}^{\text {new }}$ and $D^{\text {new }}$ into Equation 2 and solving the quadratic equation, we calculate $t_{\text {update }}$ for various $D$, i.e. the time when the next update is needed for each distance $D$. More details are provided at [10].

In the following we consider the possible states and the subsequent state changes separately:

"State change I-II" represents the scenario when the last update is received in warning "off" state. The worst case assumption that FV needs to assume, in order to prevent false negatives, as depicted in Fig. 3c), is that the speed of LV might decrease by a rate of $a_{L}^{\prime}$. False positives are less strict in this state, cf. Figure $3 b$ ). That is why, for this case, we consider the more strict requirement of false negative.

The results for various distances $D$ are depicted in Fig. 4 and can be interpreted as follows: at each inter-vehicle distance, on the $\mathrm{x}$-axis, the time $t_{\text {update }}$, on the $\mathrm{y}$-axis, depicts how long the vehicles can change their speed, in realistic boundaries, and still be in the same state as at the time of reception of the last update. After $t_{\text {update }}$ there is a chance that FV will change its state, and Eq. 2 is true, so the next update is needed to avoid false warnings. For readability we assume $t_{R}, t_{\text {sys }}$ and $D_{S}=0$. Naturally, the closer the FV gets to the critical distance the more frequently it needs a next update to make sure that the current state is identified correctly. The required update frequency increases to infinity if correct classification is desired at a very close distances (e.g., millimeter precision). This is rather unrealistic to fulfill and not even necessary (as will be discussed in the next sections).

"State change II-III" represents the scenario when the last update is received in warning "on" state and the warning was already issued at $D_{W}$. The FCW determines when the FV needs an automatic braking if the FV's driver does not respond to a warning by braking. The "State change II-III" is similar to "State change I-II", but the $D_{A B}$ is used as an orientation distance rather than $D_{W}$. The automatic braking distance $D_{A B}$ is calculated as in Eq. 1, with $t_{R}$ equals to zero and $a_{F}$ equals to the maximum achievable deceleration of the FV.

"State change II-I" represents the scenario when the last update is received in warning "on" state and the warning was already issued at $D_{W}$. The FCW determines when the warning will no longer be needed, in case that FV will brake hard enough and will change from warning "on" state back to a warning "off" state. The FV should again assume the worst case, i.e., that LV's speed might increase as FV brakes. 


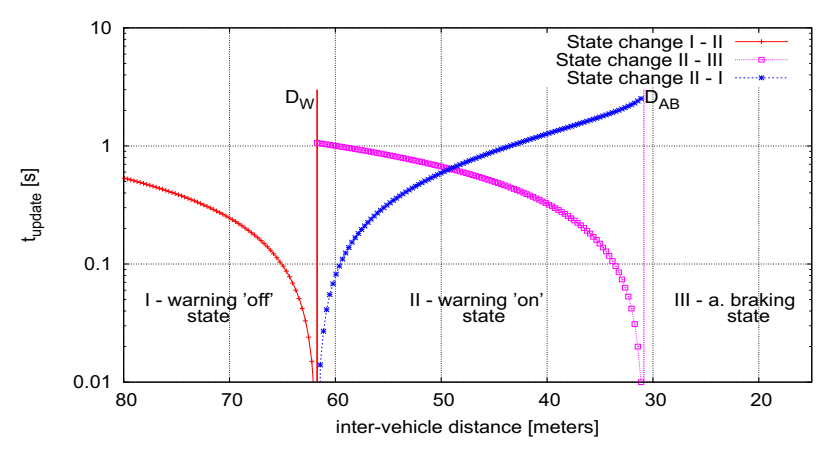

Fig. 4. Time $t_{\text {update }}$ when vehicle FV needs to receive the next update from LV for zero false positive and zero false negative. Difference between $v_{F}$ and $v_{L}$ is $\Delta v=22.22 \mathrm{~m} / \mathrm{s}(80 \mathrm{~km} / \mathrm{h})$, worst case assumption for a $v_{L}$ change rate is $a_{L}^{\prime}=-2 \mathrm{~m} / \mathrm{s}^{2}$. For $D_{W}, a_{F}=-4 \mathrm{~m} / \mathrm{s}^{2}$, for $D_{A B}$, $a_{F}=-8 m / s^{2}, t_{R}, t_{s y s}, D_{S}=0$.

State III: represents the scenario when the last update is received in automatic braking state. The FCW system of the FV is already braking with the maximum achievable deceleration. From this point on DENMs are sent out. Any realistic acceleration of the $\mathrm{LV}$, under this setting, will not lead to the case of FV going back to the warning "on" state. If the FCW application assumes softer deceleration values for an automatic braking state, it might happen that FV will leave the automatic braking state and go back to the warning "on" state. The time for the next update can then be calculated, in analogy, as going back to the warning "off" state - "State change II-I".

Obviously, the most critical areas, where the updates are needed frequently, are around the two critical distances of $D_{W}$ and $D_{A B}$, when there is a possibility to change the states. After combining the results for different states, the minimum $t_{\text {update }}$ until the next update is a requirement of the FCW application with zero false positives and zero false negatives.

\section{APpliCATION REQUiREMENT ON $t_{\text {update }}$ UNDER REALISTIC TRAFFIC CONDITIONS}

In the previous section we identified the application requirement $t_{\text {update }}$ which is determined as the time when a next update is needed based on, primarily, speed difference $\Delta v=v_{F}-v_{L}$ between both vehicles and the rate $a_{L}^{\prime}$ by which speed of LV could change. In the following, we investigate how $t_{\text {update }}$ is influenced by $\Delta v$ and $a_{L}^{\prime}$. In contrast to Section IV, we now relax the requirement of zero false positive and zero false negative and specify a desired tolerance region. The tolerance region is a geographical region prior to the distance at which the state change should occur (e.g., $\left.D_{W}\right)$. Thus, one border of the tolerance region is adjacent to e.g., $D_{W}$. The other border is a geographical distance at which the application maintains the required $t_{\text {update }}$ and at which the current FCW state is identified correctly. The warning (or automatic braking) can be triggered during this tolerance region, which allows possibility for false positive or false negative, but makes the solution feasible. We show results for exemplary tolerance regions of $5 \mathrm{~m}$ and $10 \mathrm{~m}$. Note, for large $\Delta v>10 \mathrm{~m} / \mathrm{s}$ it is not necessary to expect successful

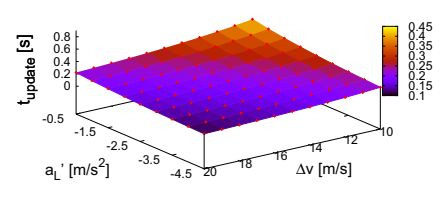

(a) Tolerance region of 5 meters

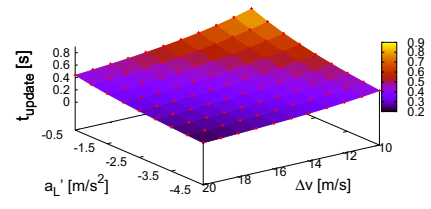

(b) Tolerance region of 10 meters
Fig. 5. The required $t_{\text {update }}$ after which the next update needs to be received for various tolerance constraints. The scenario is "State change I-II".

operation of FCW with a small tolerance $<1 m$ - no adequate driver wish to drive close to the front vehicle with a large speed difference. Whereas for small $\Delta v<10 \mathrm{~m} / \mathrm{s}$, smaller tolerance regions are realistic (vehicles moving with similar speed tend to drive closer to each other) and even desirable. We present results only for "State change I-II", other states as well as more tolerance values are presented on our website [10].

In case of "State change I-II" the FCW of the FV is in warning "off" state, i.e., the situation is safe. The smaller the speed difference $\Delta v$ the longer it might take for FV to change to warning "on" state. Fig. 5 presents how $t_{\text {update }}$ varies for different $\Delta v$ and $a_{L}{ }^{\prime}$. The shown ranges for $\Delta v$ and $a_{L}^{\prime}$, in our opinion, represent the most interesting and realistic configurations. The $\Delta v<10 \mathrm{~m} / \mathrm{s}$ represents less problematic scenarios, as vehicles drive with similar velocities, whereas $\Delta v>20 \mathrm{~m} / \mathrm{s}$ represents rather seldom or unrealistic cases for state I. For the assumed speed change rate of $\mathrm{LV}$, values do not exceed $a_{L}^{\prime}=-4.5 \mathrm{~m} / \mathrm{s}^{2}$ as it is assumed that high deceleration values should trigger transmission of DENMs.

Different tolerance regions in Fig. 5 indicate the region prior to the $D_{W}$ : tolerance region of $5 \mathrm{~m}$ corresponds to the region between $D_{W}$ and $D_{W}+5 \mathrm{~m}$ and tolerance region of $10 \mathrm{~m}$ accordingly. If we choose a tolerance region of $10 \mathrm{~m}$ and afterwards no update is received, there is a chance that by giving the warning at the initially calculated $D_{W}$ (or within the tolerance region), the system may commit a false warning, as the actual $D_{W}$ could be shifted by $\approx 5 \mathrm{~m}$ in the worst case (for $\Delta v=20 \mathrm{~m} / \mathrm{s}$ and $a_{L}{ }^{\prime}=-4.5 \mathrm{~m} / \mathrm{s}^{2}$ ). It is to be investigated, whether receiving a warning $5 \mathrm{~m}$ earlier or later could be perceived as unnecessary or too late by a driver.

It is out of scope of this paper to find the exact reaction times of the drivers, but rather to quantify its possible impact on the application requirements. If we introduce a reaction time of $t_{R}=1 s$, then the $D_{W}$ will be increased by $\Delta v \cdot t_{R}$ (similarly, the $t_{\text {sys }}$ and $D_{S}$ will increase $D_{W}$ ) and $t_{\text {update }}$ will be decreased, on average, by $10-30 \%$ depending on $\Delta v$.

\section{IMPLICATIONS UNDER REALISTIC COMMUNICATION CONDITIONS}

In order to investigate to which degree realistic communication conditions can support the application requirements we quantify the maximum number of communicating vehicles that can be supported by the FCW application. Naturally, there is a trade off between scalability and achieving close to zero false positive and zero false negative rates. 


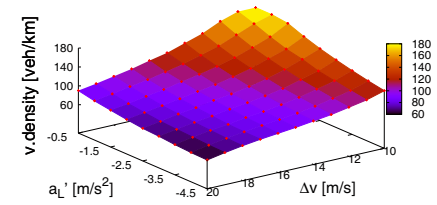

(a) Tolerance region of 5 meters

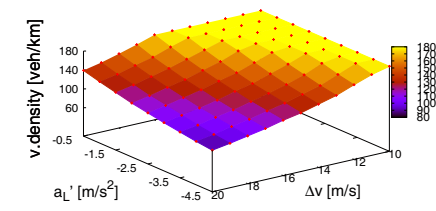

(b) Tolerance region of 10 meters
Fig. 6. Maximum vehicle density that could satisfy the application requirements for the case "State change I-II" for two tolerance constraints.

In [11] the assumption is made that applications can express their requirements in the form of awareness parameters, i.e., receiving at least $n$ packets in a time window $T$, at a certain awareness range with high probability. Then, the evaluation was performed to establish to which extent supporting these requirements means supporting traffic safety, i.e., identifying the percentage of drivers that could avoid a collision. Nevertheless, it was still unclear what are the actual application requirements and whether they can be represented in the form of awareness parameters. In the current paper, we first looked from application perspective to identify what does an application require. The identified application requirements are expressed in the form of minimum distance at which vehicles should communicate with each other, i.e., warning distance, and maximum time until the reception of the next update, i.e., $t_{\text {update }}$. These parameters perfectly match to the awareness parameters investigated in [11]. We set the number of required packets $n=1$, and the $T_{\text {window }}=t_{\text {update }}$. As FCW is a safety application we choose the probability of receiving $\mathrm{n}$ packets in time window $\mathrm{T}$ to be $\geq 99.99 \%$. The awareness range, at which the beforementioned parameters have to be fulfilled, is the warning distance $D_{W}$. To calculate probability of packet reception in realistic conditions we use the empirical model. For more details regarding the empirical model and its limitations cf. [12].

We consider the same scenario as used in Fig. 5 - "State change I-II". Thus, vehicles need to communicate at the distance of minimum $D_{W}$ with the corresponding $t_{\text {update }}$ interval(tolerance region of 5 or $10 \mathrm{~m}$ increases the required minimum communication distance accordingly). We limit the transmission range, exemplary, to $500 \mathrm{~m}$ to account for fading conditions and make sure that at least $\mathrm{n}$ packets are received at $D_{W}$. We limit the transmission rate to $10 \mathrm{~Hz}$, as the minimum $t_{\text {update }}$ is $\approx 0.1 \mathrm{~s}$, cf. Fig. 5(a) and 5(b). The maximum vehicle density that supports the application requirement $t_{\text {update }}$ in the given $\Delta v$ and $a_{L}{ }^{\prime}$ ranges is shown in Fig. 6. Notice that relaxing the tolerance increases the number of vehicles that can simultaneously participate and fulfill the FCW application requirements. For example, if we relax the tolerance from $5 \mathrm{~m}$ to $10 \mathrm{~m}$, vehicle density, achieved for $\Delta v=20 \mathrm{~m} / \mathrm{s}$ and $a_{L}{ }^{\prime}=-4.5 \mathrm{~m} / \mathrm{s}^{2}$, increases from 61 to $88 \mathrm{veh} / \mathrm{km}$.

The warning distance $D_{W}$ represents the minimum necessary communication range. Due to the wireless communication nature, the communication at distances smaller than $D_{W}$ is typically better than what is aimed for at $D_{W}$. Thus, satisfying the requirements for the "State change I-II" ( $t_{\text {update }}$ is the smallest), we satisfy the requirements for the "State change II-III" and "State change II-I".

\section{SUMMARY AND OUTLOOK}

We presented a method to analyze requirements of a Forward Collision Warning application, based on periodic updates, with zero false positive and zero false negative constraints. The application requirements are first analyzed under idealistic assumptions and expressed as the minimum communication distance and the time at which a new update is required. It has been shown that it is not always possible nor meaningful to fulfill these requirements under realistic traffic and communication conditions. We quantified the maximum number of vehicles, under realistic communication conditions, that can be supported by this FCW application. By increasing the geographical tolerance region the solution space can be increased, in terms of increasing maximum number of communicating vehicles which are supported by FCW. As a future work, a more detailed evaluation on the trade off between scalability and accuracy supported by communication system should be performed.

\section{ACKNOWLEDGEMENT}

Natalya An acknowledges the support of the Ministry of Science, Research and the Arts of Baden-Württemberg (Az: Zu 33-827.377/19,20) and the Klaus Tschira Stiftung for the junior research group on Traffic Telematics.

\section{REFERENCES}

[1] ETSI TC ITS. Intelligent Transport Systems; Vehicular Communications; Basic Set of Applications. ETSI TR 102638 V1.1.1, June 2009.

[2] F. Bai and H. Krishnan, "Reliability Analysis of DSRC Wireless Communication for Vehicle Safety Applications," in Proceedings of the IEEE Intelligent Transportation Systems Conference, Canada, 2006.

[3] M. Sepulcre and J. Gozalvez, "On the Importance of Application Requirements in Cooperative Vehicular Communications," in Proceedings of the Eighth International Conference on Wireless On-Demand Network Systems and Services, Italy, 2011.

[4] B. Kloiber et al., "Performance of CAM based Safety Applications using ITS-G5A MAC in High Dense Scenarios," in Proc. of the IEEE Intelligent Vehicles Symposium, Germany, 2011.

[5] H. Lu and C. Poellabauer, "Analysis of Application-Specific Broadcast Reliability for Vehicle Safety Communications," in Proc. of the Eight ACM Intern. Workshop on VehiculAr Inter-NETworking, USA, 2011.

[6] S. Kaul et al., "Minimizing Age of Information in Vehicular Networks," in Proc. of the 8th IEEE Comm. Society Conference on Sensor, Mesh and Ad Hoc Comm. and Networks, USA, 2011.

[7] R. Schmidt, T. Leinmueller, E. Schoch, F. Kargl, and G. Schaefer, "Exploration of Adaptive Beaconing for Efficient Intervehicle Safety Communication," IEEE Network, vol. 24, no. 1, Jan. 2010.

[8] F. Ahmed-Zaid, H. Krishnan, M. Maile, and et al., "Vehicle Safety Communications - Applications: System Design and Objective Testing Results," SAE Int. J. Passeng. Cars - Mech. Syst. 4(1):417-434, 2011.

[9] U.S. Department of Transportation and National Highway Traffic Safety Administration. Vehicle Safety Communications - Applications (VSCA). Final Report: Appendix Volume 1 System Design and Objective Test. DOT HS 811 492B. September 2011.

[10] N. An, a website with more details and plots complementing this paper http://dsn.tm.kit.edu/download/wons2013.

[11] N. An, T. Gaugel, and H. Hartenstein, "Is 95\% Probability of Packet Reception safe?" in Proceedings of the 11th International Conference on Intelligent Transport Systems Telecommunications, Russia, 2011.

[12] M. Killat and H. Hartenstein, "An Empirical Model for Probability of Packet Reception in Vehicular Ad Hoc Networks," EURASIP Journal on Wireless Communications and Networking, 2009. 